\title{
CFD Analysis to explain the Operating range extension observed during Operation in Co-rotating Mode of a Twin- impeller Centrifugal Compressor
}

\author{
Van-Thang Nguyen ${ }^{1,2^{*}}$, , Amelie Danlos ${ }^{I}$, Florent Ravelet $^{l}$, Michael Deligant ${ }^{l}$, Moises Solis ${ }^{l}$, Sofiane Khelladi $^{l}$, and \\ Farid Bakir $^{l}$ \\ ${ }^{1}$ Arts et Métiers Institute of Technology, CNAM, LIFSE, HESAM University, F-75013 Paris, France \\ ${ }^{2}$ Faculty of Vehicle and Energy Engineering, Le Quy Don Technical University, 11917 Hanoi, Vietnam
}

\begin{abstract}
Centrifugal compressors are widely used in many industrial applications because of their advantages. However, these turbomachines suffer at a low-flow rate from instabilities, such as rotating stall and surge. That leads to operational failure, pressure fluctuations and vibrations of the thorough system. Many mechanical solutions minimize these instabilities and expand the operating range towards low-flow rates like active control of the flow path, variable inlet guide vane and casing treatment. Currently, our team has developed a new compressor composed of a twin-impeller powered by autonomous systems. We notice the performance improvement and instabilities suppression of this compressor experimentally. In this paper, a CFD study is presented to explain some of these experimental observations by analyzing the inside of twin-impeller, the flow structures and thermodynamic characteristics at low flow rates operating in a corotating mode. Numerical results and experimental measurements of compressor maps are consistent.
\end{abstract}

\section{Introduction}

Use Surge line and choke line always limit operating range of compressors. Choke is an unstable state that occurs if the discharge pressure is low, and the flow rate is large. This leads to an increase in the flow velocity in the compressor. If the velocity reaches the speed of sound at the blade throat, the compressor then blocks the flow, consequently, high-frequency vibrations with small amplitudes in blades and stator vanes are generated which means the flow channels between blades suffer a blockage effect. The compressor operating in this state presents low performance and failure due to the material's fatigue effect over a long time. However, choke is more likely to occur in axial compressors than in centrifugal compressors because of the more considerable distance between the surge and choke limits of centrifugal compressors on the performance map. Therefore, a choking phenomenon in centrifugal compressors is often overlooked and it does not cause deep concern for researchers. Surge phenomenon is characterized by a strong backflow in the compressor. The pressure drops rapidly because the generated pressure does not overcome the downstream pressure gradient when the machine flow reduces below a certain minimum value. And then, the pressure and mass flow gradually increase. The equilibrium of the compressor is then re-established. This process is periodically repeated and formed a pressure oscillation cycle in the system. This oscillation creates strong impact pulses. Several internal components damage will occur if the compressor works for a long time in this state. Therefore, researchers focus on improving the surge limit to extend the compressor operating range towards low flow rates.

Indeed, all compressors are controlled to avoid surge phenomenon to ensure safety. Compared to the choking phenomenon, the surge is easier to detect thanks to the pressure pulse signals in the system. As a result, solutions have been implemented to limit the occurrence of surge phenomenon such as using safety margin by selecting a control line that is far away from the actual surge line [1,2], or using a variable inlet guide valve to allow the adjustment of inlet flow angle, one of the causes of surge [3]. The other methods were developed to suspend the surge phenomenon such as using the casing treatment method by adding grooves, slots, or holes in the casing of an inducer $[4,5,6]$, active control method uses a plenum bleed valve and air injection method to minimize flow turbulence $[7,8]$. These methods can improve the surge margin from $4 \%$ to $18 \%$. This study introduces a novel generation of centrifugal compressors that use twin-impeller to improve surge margin based on the active control method. This compressor can operate in two modes: contra-rotating mode (the two impellers rotate in the opposite direction) and co-rotating mode (the two impellers rotate in the same direction).

The measurements obtained with our experimental device reproduce the characteristics of a single-impeller centrifugal compressor, design with the same size, by varying the speed of each impeller independently [9].

* Corresponding author: thang.nguyen@ensam.eu 
The combination of the rotation speed of the upstream impeller and downstream impeller can improve the surge margin in comparison with the single impeller compressor as shown in figure 1. Especially, by changing the rotational direction of the upstream rotor (in co-rotating mode) with a speed of about $50 \%$, the surge margin can shift about $22 \%$ towards a lower flow rate. In this paper, we introduce CFD results to analyze the flow structure inside the compressor at three special points: Best Efficiency Point (BEP), Near Surge Point (NSP), and Extended Stability Point (ESP)

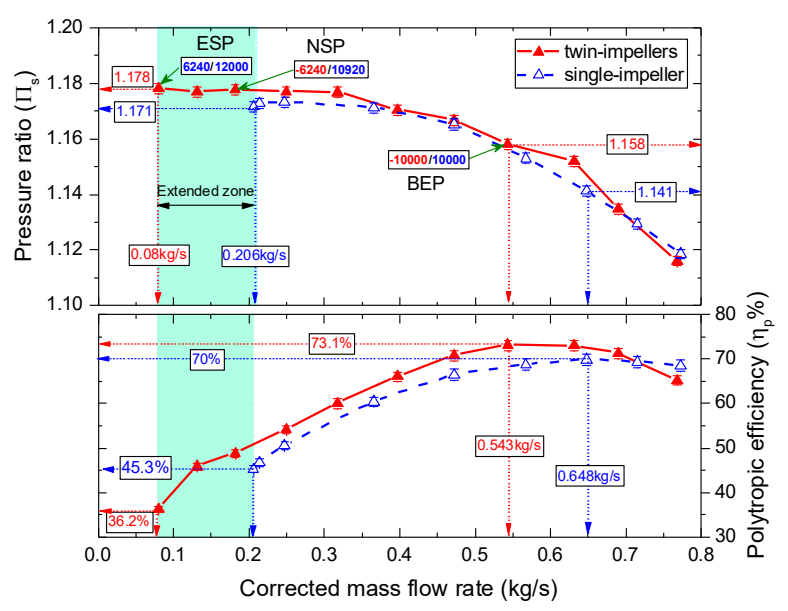

Fig. 1. Experimental characteristics of twin-impeller (red solid line) compared to single-impeller centrifugal compressor (blue dash line) at the rotation speed of $11000 \mathrm{rpm}$.

\section{CFD Model}

Computational Fluid Dynamics (CFD) plays an important role in the design and performance evaluation of turbomachinery. In this study, the twin-impeller centrifugal compressor has been simulated threedimensionally using the commercial CFD software Star $\mathrm{CCM}+13.06$. The compressor was modelled into seven domains: inlet pipe, inlet elbow, upstream-impeller, downstream-impeller, cavity, volute, and outlet pipe (Figure 2). The inlet and outlet pipe domains were extended to 5-diameter-long to reduce the effect of boundary conditions [10]. Polyhedral grids are used to discrete because of higher accuracy when the second flow is relevant [11]. In addition, a prism layer mesh was applied to increase the near-wall region to obtain the $y^{+}$ value close to the unity on the blade surface of the impellers. The details of the mesh model are shown in figure 2.

Model parameters: The simulation has been conducted in the steady-state and unsteady state, segregated implicit solver, ideal gas model. The k- $\omega$ SST turbulence model has been applied, the secondorder discretization in space has been used. In the unsteady state simulation, a time step $\mathrm{T}=2.143 \mathrm{e}-5$ was selected. The chosen value corresponds to $1.29 \mathrm{deg}$ turning of the impeller per time step. For each time step, all maximal residuals are converged to $10 \mathrm{e}-5$ after 20 internal iteration loops.

Boundary conditions: The outlet pressure was imposed as the atmospheric pressure of $101325 \mathrm{~Pa}$. The mass flow rate normal to the boundary was used as the inlet boundary condition. At the inlet, total temperature was imposed according to the value of experimental measurements. The interfaces between fluid domains are frozen rotors. The walls are adiabatic.

Mesh dependency: Choosing the right mesh model for the computation plays an essential role in saving computational resources while ensuring accuracy. Selection of the finest mesh is done through mesh independence test by constructing various mesh models. In this study, we have made three grid models to investigate the influence of mesh resolution on the performance of the compressor. More details of these mesh sets are summarized in table 1 . The simulation was performed at the speed N1 $=-11000 \mathrm{rpm}, \mathrm{N} 2=11000 \mathrm{rpm}$ and was compared to the experimental results [9]. Three performance curves corresponding to three mesh sets are shown in figure 3. It is easy to see that all models give nearly the same results however, mesh 2 and 3 produce predictive results with smaller deviation than mesh 1 . The deviation between simulation results and experimental measurements is below 5\%, which is entirely acceptable. Mesh 2 and 3 show nearly the same results in BEP and low flow regions, consequently, mesh 2 was selected to save resources and computation time.

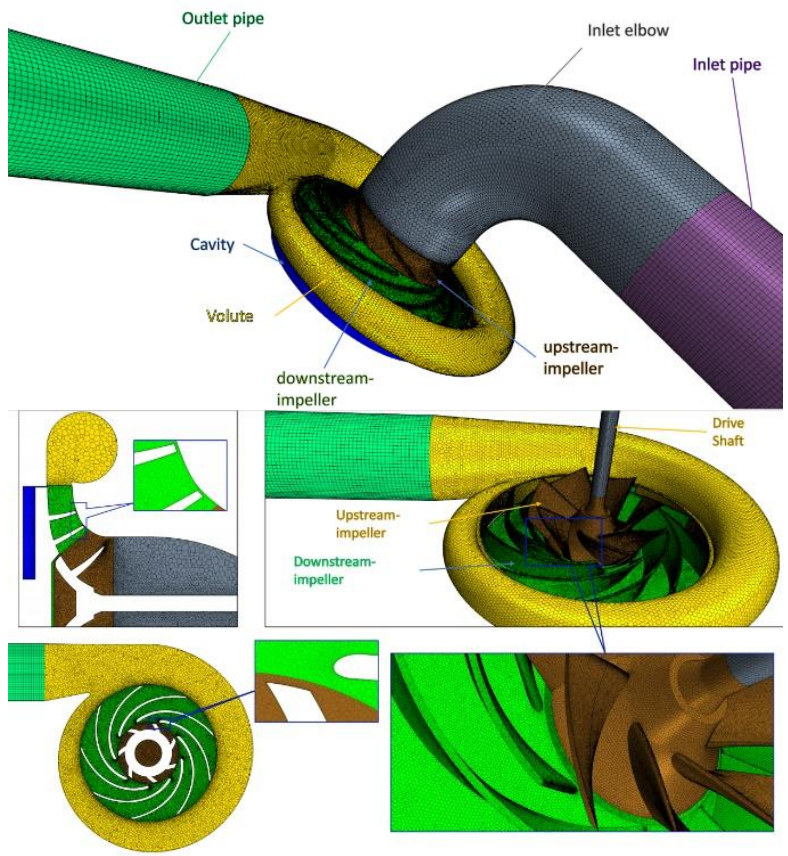

Fig. 2. Mesh details of twin-impeller centrifugal compressor.

Table 1. Details of mesh sets used for mesh sensitivity study.

\begin{tabular}{c|c|c|c}
\hline & Mesh 1 & Mesh 2 & Mesh 3 \\
\hline Inlet pipe & 74880 & 185760 & 308160 \\
\hline Inlet elbow & 97737 & 227858 & 366104 \\
\hline Impeller 1 & 309151 & 697922 & 1373753 \\
\hline Impeller 2 & 635511 & 1273090 & 2139742 \\
\hline Cavity & 188911 & 342755 & 518865 \\
\hline Volute & 70711 & 155067 & 311061 \\
\hline Outlet pipe & 74880 & 101340 & 212220 \\
\hline Total & 1430541 & 2983792 & 5229905 \\
\hline
\end{tabular}




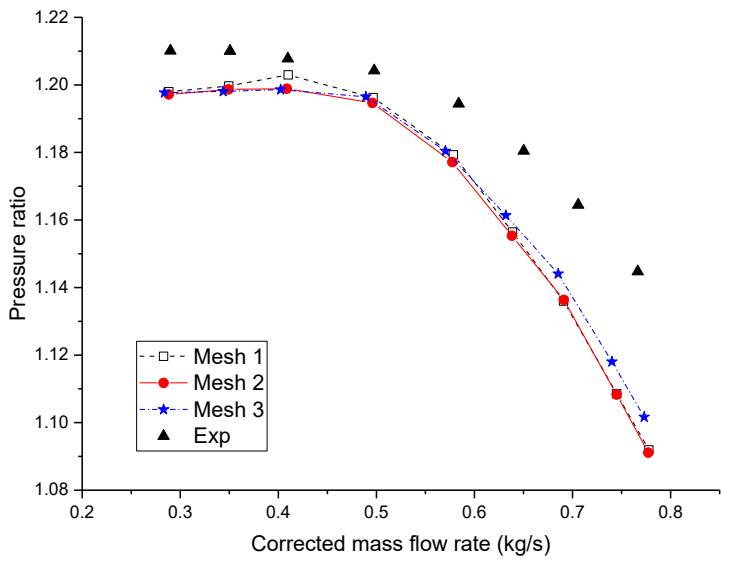

(a)

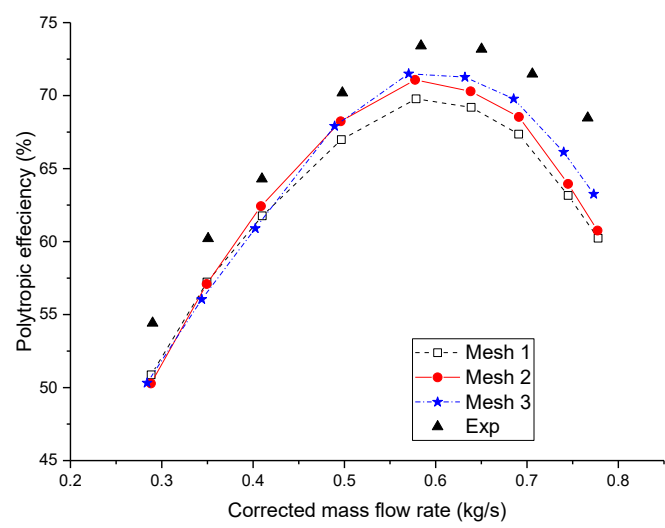

(b)

Fig. 3. Simulation results with different mesh sizes in comparison with experimental measurements at the speed of N1=-11000rpm, N2=11000rpm: Pressure ratio (a) and polytropic efficiency (b).

\section{Results and discussion}

\subsection{Steady state simulation}

The steady-state method uses the moving reference frame technique applied on impeller regions while the grid is not moving. In this technique, the Coriolis and centrifugal forces are added to the momentum equations so even though the mesh does not rotate, the impeller motion is still modelled. For the first step, a steady-state simulation has been performed to analyze the flow structure inside of twin-impeller compressor. Three points: BEP, NSP, ESP have been simulated with the working parameters based on experimental results and listed in Table 2.

Table 2. Operating parameters of three special points: BEP, NSP, ESP

\begin{tabular}{c|c:c:c}
\hline & BEP & NSP & ESP \\
\hline Mass flow rate $(\mathrm{kg} / \mathrm{s})$ & 0.4568 & 0.15 & 0.066 \\
\hline $\mathrm{N}_{1}(\mathrm{rpm})$ & -10000 & -6420 & 6240 \\
\hline $\mathrm{N}_{2}(\mathrm{rpm})$ & 10000 & 10920 & 12000 \\
\hline
\end{tabular}

\subsubsection{Flow analysis}

Figure 4 shows the pressure distribution inside the compressor on an equipotential surface at $0.5 \mathrm{span}$. At $\mathrm{BEP}$, the pressure increases gradually from the leading edge to the trailing edge of each impeller and from the volute tongue to the exit cone region.

At NSP, it is easy to see the large differential pressure between the exit cone region and the inlet volute tongue. Especially, a local high-pressure region appears at the pressure surface of the downstream impeller. The cause of this phenomenon is that these blades face the backward pressure gradient from the exit cone. It is being developed inside the volute due to the decrease in compressor flow. If the mass flow of the compressor continues to decrease, a back and strong flow is generated. Consequently, the compressor falls into the surge phenomenon. In order to help the compressor to operate at a lower flow rate than NSP with the same pressure ratio, the speeds of the two impellers had to change. Specifically, the speed of the upstream impeller decreases and the speed of the downstream impeller increases intending to modify the inlet condition of each impeller. ESP is the working limit point of the compressor, at which the upstream impeller rotates in a similar direction as the downstream impeller (in co-rotating mode). In this way, the compressor can operate stably at very low flow rates while keeping the compression ratio unchanged. It can be noted that the pressure distribution at ESP is virtually identical to NSP. However, the local pressure area on the downstream-impeller pressure surface is higher due to the impact of the fluid flowing out from the adjacent blade passage.

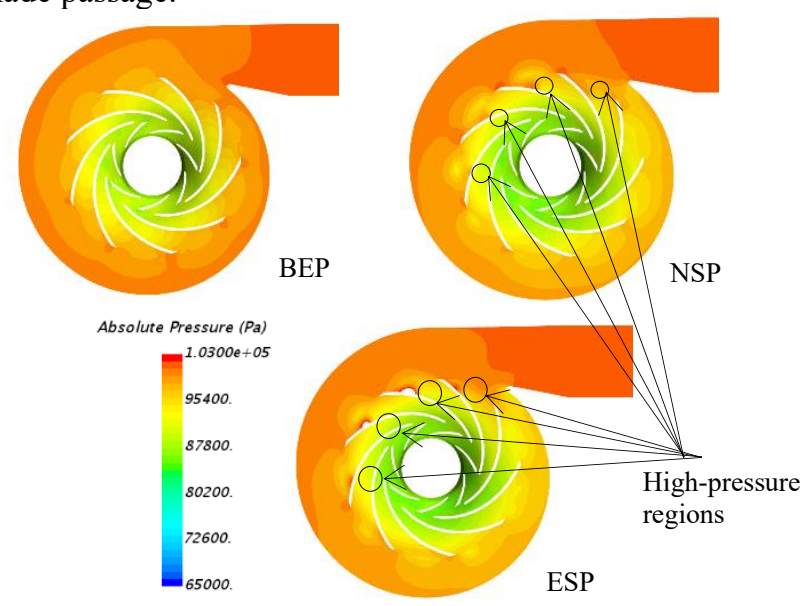

Fig. 4. Pressure distribution on an equipotential surface at 0.5 span of three operating points

Figure 5 shows the static pressure distribution on a pressure side (PS) and a suction side (SS) of the two impellers along the streamwise direction from the leading edge to the trailing edge. It can be seen the pressure difference between the PS and SS of both impellers. This shows that the flow in the blade passage is homogeneous, and the compressor works with the highest efficiency. However, there is a difference in the pressure distribution between the two impellers at NSP. The upstream impeller still has a clear distinction 
between PS and SS; meanwhile, the downstream impeller has a small pressure difference between these two sides, which means the turbulence appeared in the blade passage. When the compressor operates at a lower flow rate with a co-rotating mode (ESP), the upstream impeller has no pressure difference between PS and SS. It can be noted that the pressure at the trailing edge slightly decreases relative to the leading edge. This shows that the co-rotating effect changes the working condition slightly at the leading edge of the downstream-impeller and makes it better compatible with a low flow rate in case of dropped flow rate, and then limits the occurrence of the surge.

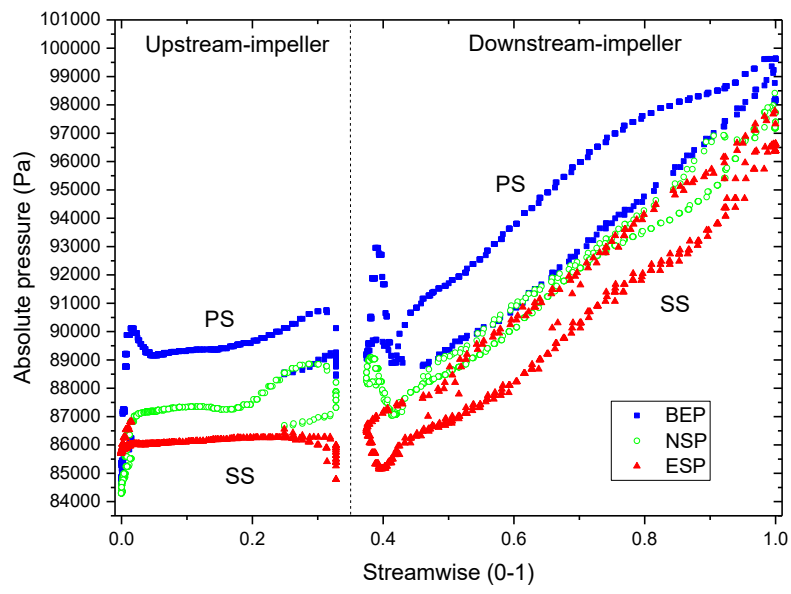

Fig. 5. Blade loading at $0.5 \mathrm{span}$ of three operating points

Figure 6 shows the relative velocity distribution on the equipotential surface at 0.5 span. At BEP, the velocity distribution is uniform within the impellers as well as inside the volute. Furthermore, jet/wake patterns can be clearly observed at the downstream impeller exit. Furthermore, two different velocity regions can be seen at the downstream-impeller blade exit. The high velocity pattern appears on the pressure side and a lower velocity pattern is shown on the suction side of the impeller. The variation of discharge flow velocity at the impeller exit has been tested and demonstrates the existence of a jet/wake flow pattern by Eckardt [12]. However, NSP illustrates the turbulence within the blade passage close to the volute tongue. It develops in the opposite direction of the impeller rotation. Because of the pressure gradient from the exit cone, the fluid flow is restricted at the exit blade. The vortices formed within the blade passage create localized high-pressure zones on the pressure surface of the blade as described in the pressure distribution section. These eddies completely blocked four downstream impeller blade passages while the flow can circulate normally in the remaining blade passages. This shows that the compressor is still operating in a steady state but close to surge. Almost like the NSP, eddies have appeared in both impellers of the ESP. Although eddies appear in most blade passages and obstruct the fluid flow there, the fluid flow still passes through some other blade passages thanks to the corotating effect of the upstream impeller. This effect helps to change the working conditions at the impeller inlet thereby reducing the incidence angle, which causes eddies within the blade passage. Therefore, the compressor still operates stably.

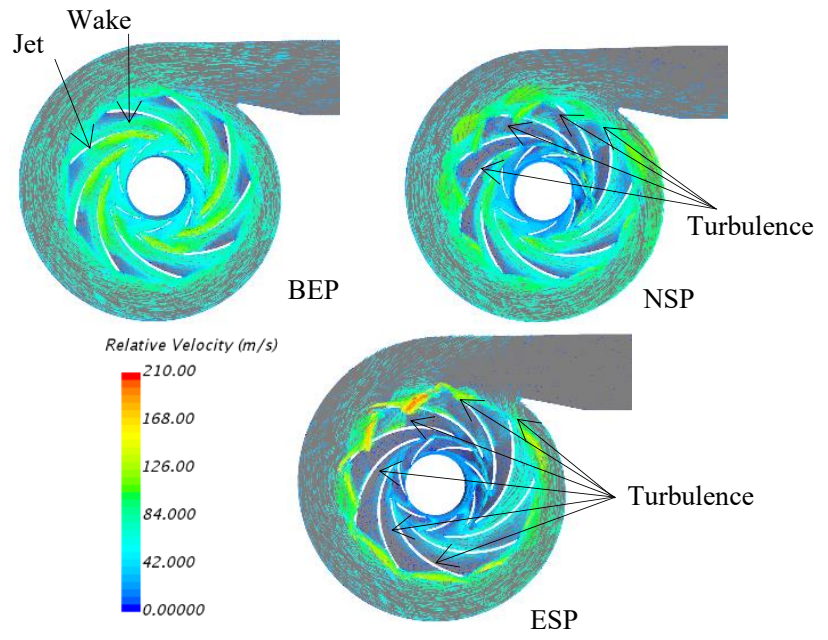

Fig. 6. Relative velocity vector distribution on an equipotential surface at 0.5 span for three operating points

The presence of turbulence within the blade passages at NSP and ESP rapidly reduces the compressor performance due to increased losses. The eddy regions increase entropy within the impellers. This increase can be seen clearly at ESP as shown in Figure 7. At BEP, the entropy distribution is uniform, which shows that the compressor operates at the highest efficiency and least loss. The uneven distribution of entropy within the downstream impeller of NSP and ESP due to eddies increases entropy rapidly inside the blade passage. The most increase of entropy can be observed inside the blade passage near the volute tongue of ESP, where the entropy value is tripled compared to the BEP. This shows that the compressor operating at this point suffers many losses, so its performance is low. This is completely consistent with the performance curve shown in Figure 1.

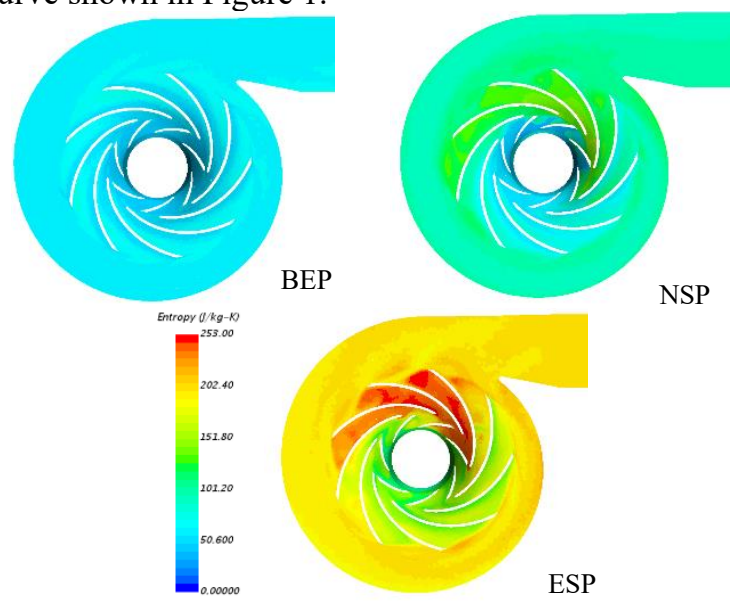

Fig. 7. Entropy distribution on an equipotential surface at 0.5 span of three operating points

\subsubsection{Asymmetry effect}

Due to the unique structure of twin-impeller compressors, the inducer must use an inlet elbow so that the inlet becomes asymmetric. This also affects the compressor performance as well as the surge limit point. Figure 8 compares the flow structure on the inlet elbow 
cross-section of the three selected operating points. The inducer asymmetry does not seem to have a significant effect on the inlet flow structure at BEP. However, the effect of asymmetry at NSP and ESP is clearly visible because of the decrease in mass flow. At these points, the downstream pressure gradient increase leads to make a reserved flow at the impeller tip. In addition, the change in pressure from the small to the large radius sides due to the asymmetry of the elbow combined with the reserved flow creates vortex regions with different sizes on both sides of the inducer. The size of the vortex increases as the flow decreases. It can develop about $50 \%$ of the area of a large radius at ESP. The asymmetry inlet causes the surge to appear earlier by increasing the incidence angle.

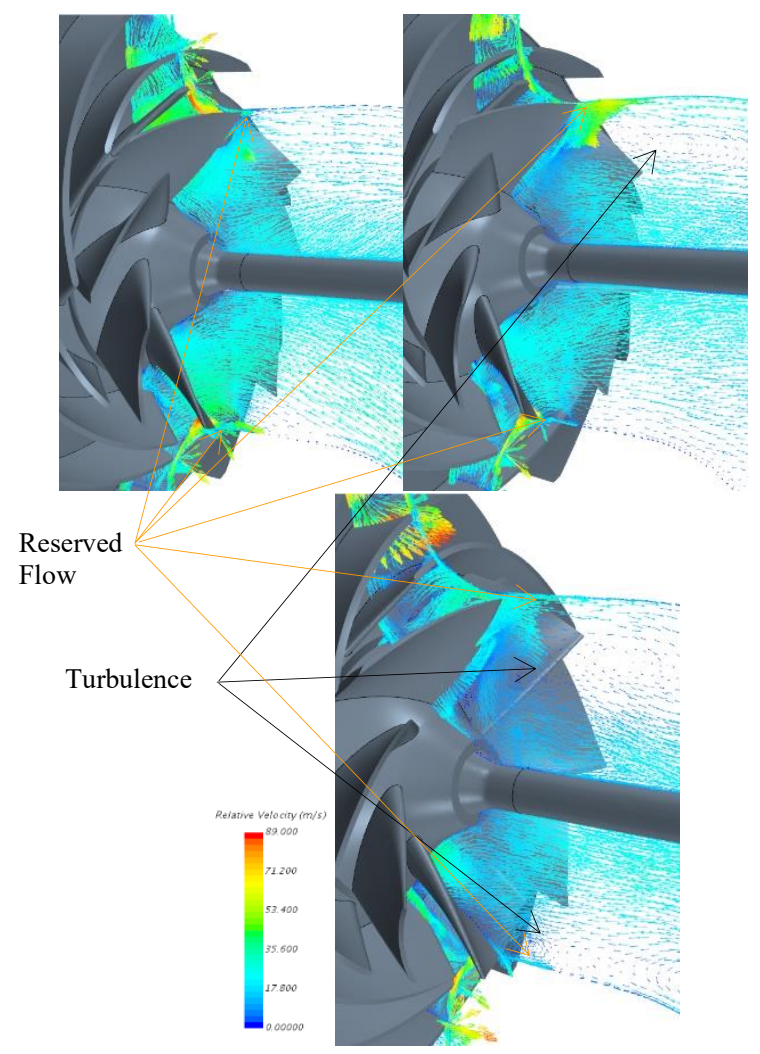

Fig. 8. Flow structure at the compressor inlet at three operating points

\subsection{Unsteady state simulation}

An important drawback of steady-state simulation is that the flow field depends on the relative position of these elements. The unsteady state simulation, on the other hand, allows the adjacent cells to slide relative to another one. The sliding interface is updated every time step and the cells do not have a correspondent on the other side of the interface, but the connectivity changes every time. In this section, the unsteady-state simulation was performed to study the change in fluid flow structure inside the compressor when the relative position of blade rows changes. This allows to observe the simulation results more closely than reality.

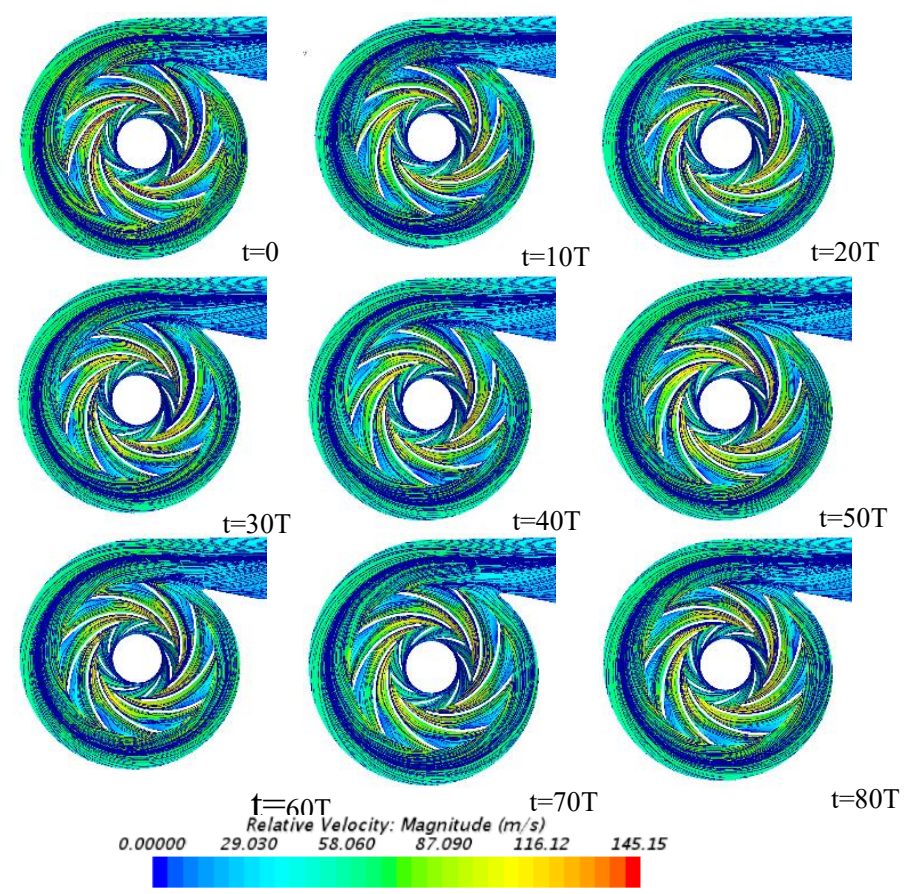

Fig. 9. Various streamline pattern according to the time-step on a half span at BEP

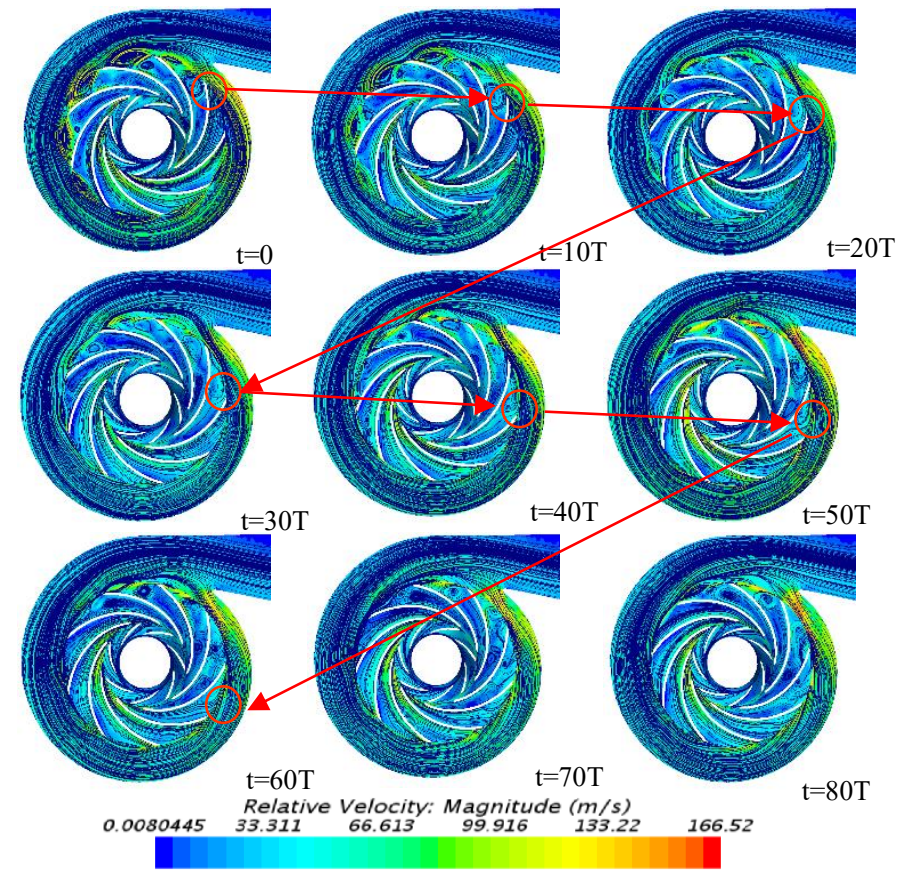

Fig. 10. Various streamline pattern according to the time-step on a half span at NSP

Figures 9, 10, and 11 show the velocity streamlines snapshots of the compressor over 80-time steps. Each snapshot corresponds to an interval of 10time steps. At BEP, the flow pattern is quite homogenous within two impellers. The change of blade rows position does not seem to affect the flow structure, so this point consistently gives the highest performance (figure 8). Conversely, it notably influences the simulation results at NSP and ESP. At NSP, the two impellers rotate in opposite directions at the speed of N1 $=-6420 \mathrm{rpm}$ and $\mathrm{N} 2=10920 \mathrm{rpm}$, respectively. The change in the flow structure within the blade passage is clearly visible. The eddy zones decrease in size over 
time steps. A vortex is marked, for example, that appears in the blade passage as shown in Figure 9. This vortex size shrinks gradually over time steps and disappears after 60-time steps. However, the simulation results over $1 \mathrm{~s}$ show that the appearance and disappearance of the vortex regions are repeated many times. This phenomenon shows that this is the limit of stability of the compressor at this rotation speed.

With co-rotating mode, twin-impeller compressors can operate at a lower flow rate than the limit flow in contrarotating mode (NSP) but still maintain a good compression ratio. Figure 10 shows the streamline structure inside the compressor at ESP in 80-time steps with the rotational speed of the two impellers respectively $\mathrm{N} 1=6240 \mathrm{rpm}$ and $\mathrm{N} 2=12000 \mathrm{rpm}$. The eddies within the blade passages change in size as the relative position between the blade rows changes. However, because the machine flow rate is low, these eddies do not disappear and eternally exist within the blade passages. In addition, some blade passages present a flow pattern quite uniform. This shows that the fluid flow is still circulating normally through these blade passages to maintain compressor stability.

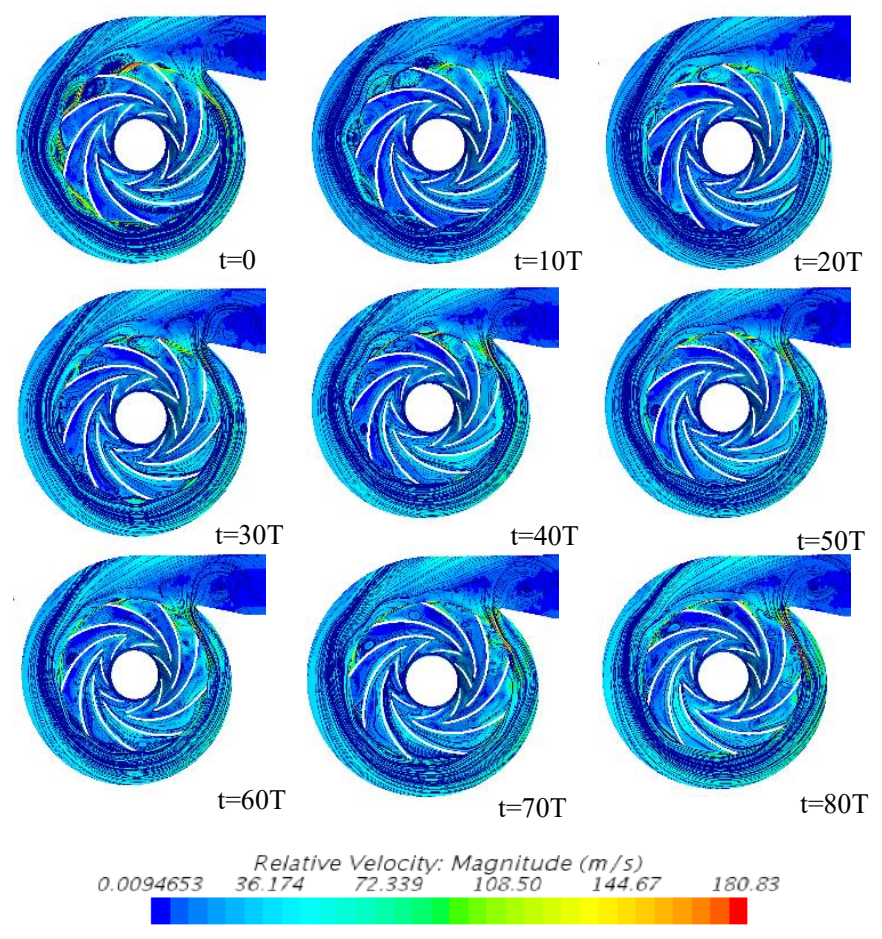

Fig. 11. Various streamline pattern according to the time-step on a half span at ESP

\section{Conclusions}

Numerical simulation studies have been conducted to further investigate the fluid flow characteristics inside the twin-impeller centrifugal compressor. Based on the experimental results, the simulation has been conducted in a steady and unsteady state. Two points in contrarotating mode are included: BEP and NSP and one point in co-rotating mode has been chosen to analyse the flow structure inside the compressor. The results indicate that:
At BEP, the flow pattern and the pressure distribution within the impellers as well as in the volute are quite homogenous. This explains why the compressor has the highest performance at this point. However, the eddies can be observed at NSP. They are localized in some blade passage of the downstream impeller near the volute tongue. The eddies decrease in size in the adjacent blade passage towards the opposite rotational direction of the downstream impeller. The blade passages are partly or completely blocked by these eddies and generate the local high-pressure region on the blade surface. The same thing happens at ESP, where more eddies appear in blade passages in both impellers. However, some blade passage still maintains a uniform flow structure that allows fluid to pass through to keep the compressor from falling into a surge state. The presence of eddy zones within the impellers increases the local entropy causing an increase in losses. Therefore, the compressor efficiency at these points is quite low.

The unsteady simulation illustrates that the change of blade row position does not affect the flow pattern at BEP. Nevertheless, it greatly influences the flow structure at NSP and ESP. According to the relative position of the blade row, the decrease in size of the eddies zone is observed at NSP. In addition, these eddies always exist within impellers at ESP. At this point, the compressor operates stably at a very low flow rate thanks to the co-rotating effect.

\section{References}

1. Botros, K. K., \& Henderson, J. F. (1994). Developments in centrifugal compressor surge Control-A technology assessment. Journal of Turbomachinery, 116(2), 240-249. https://doi.org/10.1115/1.2928358.

2. Dukle, N. \& Narayanan, K. (2003). Rotating equipment: Validating anti-surge control systems. 8. 87, 89-92, 94.

3. Simon, H., Wallmann, T., \& Mnk, T. (1987). Improvements in performance characteristics of singlestage and multistage centrifugal compressors by simultaneous adjustments of inlet guide vanes and diffuser vanes. Journal of Turbomachinery,109(1), 4147. https://doi.org/10.1115/1.3262068.

4. Koyyalamudi, V. V. N. K. S., \& Nagpurwala, Q.H. (2016). Stall margin improvement in a centrifugal compressor through inducer casing treatment. International Journal of Rotating Machinery. https://doi.org/10.1155/2016/2371524

5. Eynon, P. A., Whitfield, J. L., Firth, M.R., Perkes, A. J., \& Sexton, R. (1996). A study of the flow characteristics in the inducer bleed slot of a centrifugal compressor. ASME 1996 International Gas Turbine and Aeroengine Congress and Exhibition, GT 1996, 1. https://doi.org/10.1115/96-GT-262

6. Nikpour, Bahram (2004). Turbocharger compressor flow range improvement for future heavy duty diesel engines, Conference on thermo-and fluid dynamics processes in diesel engines, September 7th-10th, 2004, Valencia (Spain), page 125-143. 
7. Willems, F. P. T. (2000). Modelling and Bounded Feedback Stabilization of Centrifugal Compressor Surge. https://doi.org/10.6100/IR533875

8. Day, I. J. (1993). Active suppression of rotating stall and surge in axial compressors. Journal of Turbomachinery, $\quad 115(1), \quad 40-47$. https://doi.org/10.1115/1.2929216

9. Van-Thang Nguyen (2020). Experimental study of the rotor-rotor aerodynamic interaction of a counterrotating centrifugal compressor. Fluid's mechanics. PhD thesis, HESAM Université, NNT: 2020HESAE031, tel-0296704

10. Broatch, A., Galindo, J., Navarro, R., García-Tíscar, J., Daglish, A., \& Sharma, R. K. (2015). Simulations and measurements of automotive turbocharger compressor whoosh noise. Engineering Applications of Computational Fluid Mechanics, 9(1), 12-20. https://doi.org/10.1080/19942060.2015.1004788

11. Galindo, J., Hoyas, S., Fajardo, P., \& Navarro, R. (2013). Set- up analysis and optimization of CFD simulation for radial turbines. Engineering Applications of Computational Fluid Mechanics, 7(4), 441-460.

12. Eckardt, D. (1975). Instantaneous Measurements in the Jet-Wake Discharge Flow of a Centrifugal Compressor Impeller. Journal of Engineering for Power, 97(3), 337-345. https://doi.org/10.1115/1.3445999 